\title{
Análisis de una encuesta poblacional para determinar los factores asociados al control de la diabetes mellitus en México
}

\author{
Alfonso Claudio Hernández-Romieu, MC,(I) Alejandro Elnecavé-Olaiz, MC,(I) \\ Nidia Huerta-Uribe, MC,(1) Nancy Reynoso-Noverón, M en C. ${ }^{(2)}$
}

\section{Hernández-Romieu AC, Elnecavé-Olaiz A, Huerta-Uribe N, Reynoso-Noverón N. Análisis de una encuesta poblacional para determinar los factores asociados al control de la diabetes mellitus en México. Salud Publica Mex 20I 1;53:34-39.}

\section{Resumen}

Objetivo. Explorar la asociación entre recomendaciones dietéticas, ejercicio, acceso a seguridad social y medicamentos, y calidad de la atención médica con el grado de control glucémico en pacientes diabéticos. Material y métodos. La información y muestras sanguíneas se obtuvieron en 2005. Se analizó la proporción de pacientes que se encontraban en buen control ( $\leq 7 \%)$, mal control $(7.01 \%$ - 9.5\%) y descontrol severo (>9.5\%), de acuerdo con su $\mathrm{HbAl}$ c. Fueron empleados modelos de regresión logística binaria para determinar la asociación entre los factores y niveles de glucemia. Resultados. El $30 \%$ de los pacientes diabéticos se encontraban en buen control. Conclusiones. Recibir consulta con un nutriólogo disminuye la posibilidad de descontrol severo. Un alto porcentaje de los pacientes diabéticos se encuentra en alto grado de descontrol, por lo que es urgente reforzar el acceso y calidad de la atención ofrecida a estos pacientes.

Palabras clave: diabetes mellitus; Hemoglobina A Glucosilada; calidad de la atención de salud; acceso a los servicios de salud; México

\author{
Hernández-Romieu AC, Elnecavé-Olaiz A, \\ Huerta-Uribe N, Reynoso-Noverón N. \\ Analysis of population survey \\ for determining the factors associated with the control \\ diabetes mellitus in Mexico. \\ Salud Publica Mex 20I I;53:34-39.
}

\section{Abstract}

Objective. Determine the influence of nutritional counseling, exercise, access to social healthcare and drugs, and the quality of medical care on the control of diabetics. Material and Methods. The information and blood samples were obtained in 2005. Glycemic control was defined as good if $\mathrm{HbAlc}$ was $\leq 7.0 \%$, poor from $7.01 \%-9.50 \%$ and very poor if $\mathrm{HbAlc}>9.5 \%$. Binary logistic regression models were used to determine the association of these factors with $\mathrm{HbAl}>>9.5 \%$. Results. Thirty percent of the patients with a medical diagnosis of diabetes had adequate metabolic control. Conclusions. Nutritional guidance was associated with an increase in the degree of control.A majority of diabetics have poor or very poor glycemic control.Strengthening the quality of and access to medical care for these patients is urgently needed.

Key words: diabetes mellitus; Hemoglobin A, Glycosylated; quality of health care; health services accessibility; Mexico

(I) Facultad de Medicina, Universidad Nacional Autónoma de México. México DF, México

(2) Secretaría de Salud. México DF, México.

Fecha de recibido: 29 de junio de 2010 - Fecha de aceptado: 27 de octubre de 2010 Solicitud de sobretiros: M en C Nancy Reynoso Noverón. Secretaría de Salud. Lieja 7, Ier. Piso, col. Juárez. 06696 Delegación Cuauhtémoc México DF, México.

Correo electrónico:nrn231002@yahoo.com.mx 
$\mathrm{L}^{\mathrm{a}}$ diabetes mellitus (DM) es una enfermedad cuya prevalencia mundial ha incrementado en los últimos años. En México, la diabetes se ha convertido en la primera causa de muerte al contribuir con $12 \%$ del total de muertes. ${ }^{1}$ Se estimó para el año 2030 una prevalencia nacional de $10.9 \%$ y tan sólo en 2002 se registraron 114.6 nuevos casos por cada 100000 habitantes. ${ }^{2-3}$

En el ámbito socioeconómico se describen pérdidas de 264 mil años de vida saludables por muertes prematuras y 171 mil por discapacidad en diabéticos de más de 45 años. Diferentes autores indican que el costo indirecto de la diabetes en 1991 ascendió a 330 millones de dólares y el directo fue de 100 millones de dólares. ${ }^{4-7}$ Las pérdidas para los servicios de la salud son del orden de 318 millones de dólares por año; ${ }^{8}$ la atención de esta enfermedad cuesta a los sistemas de salud hasta 15\% del total de sus recursos, ${ }^{7}$ y es el rubro del gasto más importante del IMSS. ${ }^{9}$

Este costo de atención se debe principalmente a las complicaciones secundarias de la enfermedad. Es necesaria una orientación de las estrategias de intervención terapéutica para retrasar el desarrollo de daño a nivel macro y microvascular, ${ }^{6}$ para disminuir así los costos de atención personales y familiares, y evitar la pérdida de productividad individual.

Hay guías clínicas que describen las metas básicas del tratamiento otorgado al paciente diabético y el proceso de atención más eficiente a través del cual se alcanzan. ${ }^{10-14}$ La concentración de hemoglobina A glucosilada $(\mathrm{HbA1c})$ ha sido descrita en todas estas guías como un importante marcador biológico para la supervisión de los pacientes diabéticos en tratamiento. Los niveles de $\mathrm{HbA1c}$ reflejan el promedio de las concentraciones de glucosa plasmática durante los 3 o 4 meses anteriores y se emplea como una medida indirecta de la calidad del control en el que se encuentra un sujeto con DM. ${ }^{15-17}$

Los lineamientos internacionales recomiendan niveles menores a 7\% de HbA1c como un buen control glucémico debido a que niveles debajo de esta cifra han demostrado atenuar las complicaciones microvasculares y neuropáticas de la diabetes tipo 1 y $2 .{ }^{18}$ La reducción a niveles promedio de $7 \%$, comparado con cifras cerca de 7.9\% en un periodo de 10 años, con tratamiento, disminuye el riesgo de presentar cualquier complicación final de la diabetes en $12 \%$ y aminora en $25 \%$ la incidencia de enfermedades microvasculares, lo que incluye $16 \%$ en el infarto al miocardio $(\mathrm{P}=0.052) .{ }^{19-21}$

El sistema de salud mexicano se ha esforzado por la mejora en el acceso a la atención y el cumplimiento de las Guías de Manejo del Paciente Diabético, sin embargo, los estudios muestran un inadecuado control metabólico de los diabéticos en el país. Es por esto que el objetivo de este estudio es explorar la asociación de la recomen- dación de dieta y ejercicio, acceso a la seguridad social y a medicamentos, y la calidad de la atención médica con el grado de control glucémico de los diabéticos en México.

\section{Material y métodos}

La población de estudio se conformó por 937 personas autoidentificadas como diabéticas por medio de una encuesta probabilística y a las cuales se les realizó una medición de niveles de $\mathrm{HbA1c}$ en sangre. Esto fue llevado a cabo en el año 2005, en zonas rurales y urbanas de siete estados de la República mexicana.

El diseño muestral se ha presentado con detalle en otras publicaciones. ${ }^{22}$ Brevemente, la primera unidad de muestreo la constituyeron diversos conglomerados de salud, constituidos por una unidad, hospital o clínica de salud y la población que asiste a dicha unidad. Se estudiaron 100 conglomerados, en los cuales se seleccionaron 380 hogares al azar por conglomerado. En cada uno de estos hogares se aplicó la encuesta y se obtuvieron finalmente 31623 encuestas. Los residentes con diagnóstico de diabetes $(n=1844)$, contestaron el cuestionario específico. Se seleccionaron 50\% de las viviendas (190 por conglomerado) para realizar la prueba de hemoglobina A glucosilada a 937 pacientes que fueron incluidos en este análisis. El estudio incluyó conglomerados de los siguientes estados: Guerrero, Jalisco, Estado de México, Morelos, Oaxaca, San Luis Potosí y Sonora.

El cuestionario específico fue creado por personal de la Universidad de Harvard, en colaboración con la Secretaría de Salud y el Instituto Nacional de Salud Pública (INSP). El cuestionario constó de 86 reactivos en los cuales se indaga sobre antecedentes del padecimiento, dieta, uso de medicamentos, visitas al médico, vigilancia de complicaciones, determinaciones de glucosa y lípidos, evaluación y satisfacción con el tratamiento, participación en grupos de apoyo, salud mental y presencia de complicaciones. Se obtuvo el consentimiento informado de todos los participantes en la aplicación del cuestionario y la toma de muestras. El cuestionario y la metodología fueron avalados por el Comité de Ética del INSP.

Análisis de datos. Se realizó estadística descriptiva sociodemográfica de los pacientes (sexo, edad, escolaridad, tiempo de diagnóstico) además del nivel de $\mathrm{HbA1c}$, seguimiento de recomendaciones dietéticas y de actividad física, el uso de medicamentos, la atención del médico, y el acceso a la seguridad social. Se definió un índice de calidad de la atención médica, siendo adecuada si en su última consulta el médico lo pesó, midió tensión arterial, explicó la toma de medicamentos y dio recomendaciones dietéticas y de ejercicio. En caso de 
que faltara alguno de estos puntos la atención médica se consideró como inadecuada; estas variables también fueron analizadas de forma individual.

Se describieron, según las respuestas del cuestionario, las razones para no seguir dieta y no tomar medicamentos como: "no tengo dinero", "el médico dijo que no es necesario", "poca fuerza de voluntad" o "me siento bien".

De acuerdo a la concentración de HbA1c, se analizó la proporción de pacientes que se encontraban en buen control glucémico $(\leq 7.00 \%)$, mal control $(7.01 \%$ - 9.50\%) o severo descontrol ( $>9.50 \%)$. De acuerdo a esto y debido a que el $\mathrm{P}_{50}$ de los niveles de $\mathrm{HbA1c}$ fue de $9.5 \%\left(\mathrm{P}_{25}\right.$ $\left.6.85 \%-\mathrm{P}_{75} 11.3 \%\right)$, se creó una variable que definiera a los pacientes en descontrol severo ( $\mathrm{HbA1c}>9.50 \%)$ y a los sujetos "mal controlados" (HbA1c $\leq 9.50 \%)$. Mediante modelos de regresión logística binaria se determinó el efecto de las variables analizadas sobre el control glucémico. Para analizar los datos se utilizaron los programas STATA, Epi Info 6.0 y Microsoft Excel XP.

\section{Resultados}

Se obtuvo una prevalencia del padecimiento de 5.8\%. De los 937 pacientes, $65.85 \%$ eran mujeres y $34.15 \%$ hombres; la edad promedio fue de 56 años ( $D E=12$ años) en mujeres y de 57 años ( $D E=12$ años) en hombres. En cuanto a la escolaridad, 29.9\% de los participantes refirió un grado menor a la primaria completa, 55\% cursó la primaria completa y $15 \%$ reportó estudios superiores a ésta. Respecto al tiempo de diagnóstico, $10.7 \%$ recibió el diagnóstico hace menos de un año, $17.6 \%$ de 1 a 2 años atrás, $22.3 \%$ de 3 a 5 años, $21.8 \%$ de 5 a 10 años, y $27 \%$ mayor a 10 años.

El 31\% refirió no seguir las recomendaciones dietéticas: $41 \%$ por falta de voluntad y $26 \%$ por razones socioeconómicas. El $46 \%$ de los encuestados reporta no seguir las recomendaciones de ejercicio.

De la población encuestada, $85 \%$ indicó que toma medicamentos; el resto no lo hace por sentirse bien (42\%) o por falta de ingresos (32\%). Se encontró que sólo $24 \%$ cuenta con seguridad social; los derechohabientes obtuvieron un valor de $\mathrm{HbA1c}$ de $8.5 \%$, mientras que los otros presentaron en promedio $9.1 \%(p<0.05)$.

$\mathrm{Al}$ analizar la atención médica, se encontró que a 90.7\% de los pacientes se les midió la tensión arterial, se pesó a $87.1 \%$, a $78.1 \%$ se le explicó una rutina de actividad física, a $88.1 \%$ se le indicó cómo llevar una dieta adecuada y a $90.1 \%$ se le describió la forma de tomar los medicamentos; sin embargo, $40 \%$ de los médicos tratantes omitió uno o más de estos puntos al dar consulta.
El valor promedio observado para $\mathrm{HbA1c}$ fue de 9.02\% ( $\mathrm{DE}=2.4)$, con una tendencia positiva significativa $(p<0.01)$ en el porcentaje de $\mathrm{HbA} 1 \mathrm{c}$ de acuerdo con el tiempo del diagnóstico; incrementó de $8.4 \%$ en el grupo de menos de un año de diagnóstico a $9.2 \%$ en el grupo de 10 años o más. En contraste, el valor de HbA1c varió en forma de U invertida con respecto a la edad; se observó en los menores de 40 un HbA1c de 8.6\%, de 51-55 años $9.8 \%$ y $>70$ años $8.4 \%$. No se observaron diferencias en relación con el sexo, edad, escolaridad y recomendaciones de ejercicio. La media de $\mathrm{HbA1}$ c en los que siguieron recomendaciones dietéticas fue de $8.9 \%$ vs. 9.3\% en los que no lo hicieron $(p<0.05)$. El promedio de $\mathrm{HbA} 1 \mathrm{c}$ fue mayor entre las personas bajo tratamiento farmacológico $(9.5 \%)(p<0.01)$.

$\mathrm{Al}$ analizar la atención otorgada, la Hba1c fue de 9.8\% en los que no recibieron explicación del tratamiento (8.7\% del total de los encuestados) en relación con $9.2 \%$ para los que la recibieron (91.3\% de los encuestados); sin embargo, esta diferencia no fue estadísticamente significativa. Los resultados para los participantes que recibieron información sobre la dieta tampoco fueron significativos. Una variable que arrojó resultados interesantes fue la visita a un nutriólogo; aquellos participantes que acudieron al nutriólogo en los últimos 12 meses $(\mathrm{n}=94)$ tuvieron niveles de $\mathrm{HbA1c}$ significativamente menores (8.6 vs. $9.2 p<0.05$ ).

Al momento de la encuesta, 30\% de los participantes tenía concentraciones de $\mathrm{HbA} 1 \mathrm{c} \leq 7 \%$, mientras que $50 \%$ tenía niveles de $\mathrm{HbA1c}$ por encima del 9.5\%. Con el fin de modelar los predictores de control glucémico en esta población, se ajustó un modelo logístico multivariado en el cual la variable respuesta la constituyó el descontrol glucémico severo (HbA1c > 9.50); los resultados se presentan en el cuadro I. Tener un padecimiento de más de tres años de evolución se asoció con un incremento significativo en la posibilidad de tener un severo descontrol glucémico; incluso los pacientes con más de cinco años de evolución tuvieron 2.4 veces mayor probabilidad de que existiera tal descontrol $(R M=2.46$, IC $95 \%=1.42$ a 4.26). Los participantes de 66 a 70 años estuvieron menos predispuestos a estar en descontrol severo; por otro lado, tanto el acceso a la seguridad social como el acudir al médico en los últimos tres meses mostró un efecto protector moderado de cerca de 30\% $(R M=0.77, I C 95 \%=0.54$ a 1.09); el acudir a una interconsulta con un nutriólogo disminuyó significativamente la posibilidad de estar severamente descontrolado $(R M=$ 0.61, IC $95 \%=0.38$ a 0.97 ) y paradójicamente, ésta fue dos veces mayor en los pacientes con tratamiento farmacológico, $(R M=2.05$, IC $95 \%=1.29$ a 3.27$)$. 
Cuadro I

Modelo MUlTIVARIAdo de PREDICCIÓN DE DESCONTROL GLUCÉMico. MéXICO, 2005

$\begin{array}{cccc}\text { Variable } & \begin{array}{c}\text { Razón de } \\ \text { momios }\end{array} \quad \text { Valor p } & \begin{array}{c}\text { Intervalo de } \\ \text { confianza } 95 \%\end{array}\end{array}$

Tiempo de diagnóstico

\begin{tabular}{llll} 
Menos de 12 meses & 1.0 & & \\
\hline I a 2 años & 1.02 & 0.95 & $0.57-|.8|$ \\
\hline 3 a 5 años & 1.90 & 0.02 & $1 . \mid I-3.27$ \\
\hline 5 a 10 años & 2.46 & 0.00 & $1.42-4.26$ \\
\hline Más de 10 años & 2.34 & 0.00 & $1.35-4.05$
\end{tabular}

Edad en años

\begin{tabular}{llll}
18 a 40 & 1.0 & & \\
\hline $4 I$ a 45 & 1.17 & 0.61 & $0.63-2.16$ \\
\hline 46 a 50 & 1.18 & 0.58 & $0.65-2.14$ \\
\hline 51 a 55 & 1.35 & 0.32 & $0.75-2.44$ \\
\hline 56 a 60 & 0.96 & 0.88 & $0.54-1.70$ \\
\hline 61 a 65 & 0.50 & 0.03 & $0.27-0.93$ \\
\hline 66 a 70 & 0.35 & 0.00 & $0.18-0.68$ \\
\hline 71 a 99 & 0.37 & 0.00 & $0.20-0.70$
\end{tabular}

Sexo

\begin{tabular}{llll}
\multicolumn{1}{l}{ Hombre } & 1.0 & & \\
\hline \multicolumn{1}{c}{ Mujer } & 1.17 & 0.30 & $0.87-1.58$ \\
& & & \\
Toma medicamentos (sí) & 2.05 & 0.00 & $1.29-3.27$ \\
\hline Acceso a seguridad social & 0.77 & 0.14 & $0.54-1.09$ \\
\hline $\begin{array}{l}\text { Acudió al nutriólogo en los últimos } \\
\text { I2 meses }\end{array}$ & 0.61 & 0.04 & $0.38-0.97$ \\
\hline $\begin{array}{l}\text { Acudió al médico en los últimos } \\
3 \text { meses }\end{array}$ & 0.75 & 0.10 & $0.54-1.05$
\end{tabular}

Fuente: Datos tomados de la encuesta poblacional realizada por el Instituto Nacional de Salud Pública durante 2005 en los estados de Guerrero, Jalisco, Estado de México, Morelos, Oaxaca, San Luis Potosí y Sonora

\section{Discusión}

Se mostró una prevalencia de diabetes mellitus de $5.8 \%$, mucho menor de la nacional de 10.9\%; sin embargo, los datos analizados corresponden a sujetos de zonas rurales y la incidencia y prevalencia más alta se presenta en las zonas urbanas.

La media de HbA1c en esta población es de $9.2 \%$, mientras que la mediana fue de $9.5 \%$, la cual es muy alta comparada con la reportada en otras poblaciones: en Suecia ${ }^{23,24}$ las medias reportadas de HbA1c están entre 6.7 y 7\%, y Coon y Zulkowski reportaron una media de 7.43 para pacientes atendidos en zonas rurales de Estados Unidos. ${ }^{25}$ No obstante, en mediciones de los niveles de $\mathrm{HbA1c}$ en población latina de bajos recursos económicos se ha reportado que la media se encuentra en $7.7 \%,{ }^{27}$ lo cual sugiere que el cambio de estilo de vida de pacientes latinos que migran al Norte, así como la atención en salud con mejor infraestructura y mayor acceso a medicamentos, proporciona ciertas ventajas para mantener un buen control glucémico. Al analizar las diferencias de $\mathrm{HbA1c}$ entre los grupos de edad encontramos que los mayores de 70 años son el grupo etáreo que presenta el menor grado de descontrol; esto puede deberse a que los pacientes que desarrollan complicaciones mueren antes de llegar a esta edad. ElKebbi IM y Ziemer D llegaron a una conclusión similar al estudiar el grado de control glucémico en pacientes diabéticos con comorbilidades. ${ }^{18}$

Las altas concentraciones de $\mathrm{HbA1}$ c encontradas en los pacientes diabéticos de la muestra analizada orillaron a utilizar un punto de corte para descontrol severo control $>9.50 \%$, lo que conforma un grupo de sujetos "mal controlados" con concentraciones de $\mathrm{HbA} 1 \mathrm{c} \leq 9.5 \%$ cuyo punto de corte también es elevado. Sabemos que los valores en los pacientes "mal controlados" no disminuirán la sintomatología ni la probabilidad de presentar complicaciones secundarias a la enfermedad.

Las causas del descontrol glucémico son muchas y escapan al enfoque del presente trabajo; sin embargo, es pertinente mencionar la complejidad que representa para el paciente diabético llevar un tratamiento integral y un buen apego a este, debido al cambio de hábitos y costumbres. Esta enorme proporción de pacientes con ausencia de control demuestra tanto la responsabilidad de los pacientes frente a su enfermedad como el gran reto que tiene el sistema de salud para lograr que la calidad de atención médica favorezca el apego y evitar así el desarrollo de complicaciones relacionadas, para finalmente poder disminuir la carga económica al sistema de salud y a las familias.

A pesar de los requerimientos básicos en la atención de estos pacientes, se mostró que $40 \%$ de las consultas médicas omiten elementos indispensables; la falta del cumplimiento de la NOM indica una atención médica insatisfactoria en las comunidades de los estados sondeados en el estudio. Aunque no se observó significancia estadística en la mayoría de los indicadores, se mostró que seguir las recomendaciones dietéticas tiene un efecto significativo en el control glucémico tal y como la literatura médica lo ha reportado. ${ }^{21,27} \mathrm{Al}$ analizar la presencia de consejería nutricional se observó una diferencia de $0.6 \% \mathrm{HbA} 1 \mathrm{c}$ entre los pacientes que acudieron al nutriólogo y los que no, lo que confirma la importancia de un tratamiento multidisciplinario. 
Por otro lado, pese a que $85 \%$ de la población estudiada usa medicamentos, esta variable no se asoció a un mejor control. Esto quizá se deba, primero, a que el uso de medicamentos sólo está justificado cuando el paciente no se controla con dieta y ejercicio, ${ }^{28}$ y en segundo lugar, a que los pacientes no suelen acudir al médico sino hasta que empiezan a presentar las complicaciones que resultan de un descontrol metabólico; y finalmente, los pacientes que requieren tomar medicamentos tienen un tiempo de evolución más largo que aquellos que no los toman. Lo anterior resulta alarmante e indica que es urgente revisar a detalle el modelo de atención y la calidad del mismo.

El hecho de que los derechohabientes presentaron cifras de HbA1c más bajas indica, en primera instancia, que la atención médica de especialistas y la obtención de medicamentos se facilitan con la seguridad social; en segunda instancia indica la presencia de una actividad económica productiva, es decir, la existencia de diferentes posibilidades económicas y, finalmente, proyecta que la ampliación en cobertura de los servicios médicos debería llevar a un mejor control del padecimiento y podría influir en el retraso de las complicaciones.

Un estudio realizado en 2003 entre pacientes diabéticos en el Reino Unido y Estados Unidos de América, al comparar los beneficios de una cobertura médica universal (RU) con los de una que no lo es (EUA), mostró un mejor control general de la HbA1c en el primer caso. ${ }^{29}$ Los resultados anteriores nos permiten concluir que la diabetes no depende únicamente del paciente y su adaptación a la enfermedad, sino que nos obliga a enfrentar las realidades sociales de la falta de acceso a la atención médica de calidad.

Existen varias limitantes del estudio. La información se obtuvo por medio de un cuestionario cerrado, lo que permite la introducción de sesgos por parte del paciente al responder; esto puede ocasionar ya sea una omisión de datos que podrían ser relevantes en su padecimiento o la mitigación de algunos síntomas o datos acerca del tratamiento. Ya que se trata de un estudio transversal, puede ser que los pacientes no recuerden con exactitud todo lo concerniente a la encuesta y que sus respuestas pudieran haber sido modificadas por la presencia de la enfermedad. Es relevante recalcar que no se tiene información de parte de los médicos, elemento esencial para hacer un análisis completo de la situación de estos pacientes. Debido a esto, existe la posibilidad de que la asociación observada entre tratamiento y descontrol se deba a la naturaleza transversal del estudio. Finalmente, la falta de temporalidad juega un papel importante como limitante, ya que no se sabe cuándo se hace el diagnóstico de la enfermedad ni si el paciente acude al médico por sintomatología provocada por mal control. El hecho de no poder identificar si el mal control comienza antes o después de acudir al médico introduce un sesgo por ambigüedad temporal que impide verificar una asociación objetiva entre ambas variables.

A pesar de las limitaciones, una fortaleza importante es que la medición de $\mathrm{HbA1c}$ es un marcador muy confiable de control metabólico, ya que indica el grado de control en un intervalo de 120 días. Por medio de esta medición es posible evitar los sesgos de medición de glucosa en sangre.

\section{Conclusión}

Resulta alarmante la baja proporción de pacientes diabéticos controlados encontrados en este análisis que, aún teniendo básicamente una representatividad rural, demuestra que las metas del tratamiento a este grupo de pacientes aún no se han cumplido. El control de la glucemia es de causa multifactorial; se debe mejorar el acceso y la calidad con la que se atiende a este grupo de pacientes e intentar así una mejor adherencia al tratamiento y con ello lograr una menor incidencia de complicaciones secundarias a la enfermedad, padecimientos que representan una gran carga económica tanto para los sistemas de salud del país como para los pacientes y sus familias.

\section{Agradecimientos}

Se agradece al Dr. Mauricio Hernández Ávila por su asesoría y apoyo incondicional. Se agradece al Instituto Nacional de Salud Pública por su ayuda en la obtención de los datos.

Declaración de conflicto de intereses: Los autores declararon no tener conflicto de intereses.

\section{Referencias}

I. Secretaría de Salud. Mortalidad, 2002. México: SSA, 2002.

2. Secretaría de Salud.Anuario Estadístico. 2002. México: SSA, 2002.

3. Secretaría de Salud. Salud: México 2003. Información para la rendición de cuentas. México: SSA, 2004.

4. Brown JB, Pedula KL, Bakst AW. The progressive cost of complications in type 2 diabetes mellitus. Arch Intern Med 1999; 159:1873- I880.

5. Secretaría de Salud. Información para la rendición de cuentas. México: SSA, 2004.

6. Secretaría de Salud. Dirección General de Epidemiología, anuarios de Morbilidad. México: SSA, 2004

7. Lozano-Ascencio R, Frenk-Mora J, González-Block MA. El peso de la enfermedad en adultos mayores. Salud Publica Mex 1996; 38: 419-429. 8. Arredondo A, Zuñiga A. Economic consequences of epidemiological changes in diabetes in middle-income countries. The Mexican case. Diabetes Care 2004;27(I):104-109. 
9. Instituto Nacional de Salud Pública. Evaluación externa del Programa Oportunidades. México: INSP, 2004:209-245.

10. Secretaría de Salud. Norma Oficial Mexicana NOM-0 I5-SSA2-1994, para la prevención, tratamiento y control de la diabetes. México: SS, 1999. II. Grupo de Estudio de la Diabetes en Atención Primaria de la Sociedad Catalana de Medicina Familiar y Comunitaria. Diabetes mellitus tipo 2: protocolo de actuación. FMC 2000; 7 (Supl 6): I-54.

12. Secretaría de Salud Norma Oficial Mexicana NOM-015-SSA2-1994, para la prevención, tratamiento y control de la diabetes. Punto II.I I.I.3. México: SSA, 1999.

13. Nathan DM, Buse JB, Davidson MB, et al. Medical Management of Hyperglycemia in Type 2 Diabetes: A Consensus Algorithm for the Initiation and Adjustment of Therapy. Diabetes Care 2009; 32:193-203. 14. American Diabetes Association. Standards of medical care in diabetes -- 2009. Diabetes Care 2009;32:SI3-S6I.

15. Greci LS, Kailasam M, Malkani S, Katz DL, Hulinsky I,Ahmadi R, et al. Utility of $\mathrm{HbAIc}$ levels for diabetes case finding in hospitalizaed patients with hyperglycemia. Diabetes Care 2003;26(4): 1064- 1068.

16. Rohlfing CL, Wiedmeyer H, Little R, England J, Tennill A, Goldtein D. Defining the relationship between plasma glucose and $\mathrm{HbAl}$. Diabetes Care 2002; 25(2):275-278

17. Gómez-López V, García-de León S, López C. Control de la diabetes mellitus tipo 2. El índice de hiperglicemia como indicador. Rev Med IMSS 2002; 40 (4): 28I-284. [Consultado en diciembre 3 de 2008]. Disponible en: www.imss.gob.mx/NR/rdonlyres/9ED79478-352C-4F0F-9F274D766328973F/O/hiperglucemia I0.pdf 18. El-Kebbi IM, Ziemer D, Cook CB, Miller ChD, Gallina D, Phillips LD. Comorbidity and glycemic control in patients with type 2 diabetes. Arch Intern Med 200I;I6I(I0): I295-I300.

19. UK Prospective Diabetes Study Group. Quality of life in type 2 diabetic patient is affected by complications but not by intensive policies to improve blood glucose or blood pressure control (UKPDS 37). Diabetes Care 1999;22:I I25-II36.
20. Henricsson M, Nystrom L, Blohme G, Ostman J, Kullberg C, Svensson $M$, et al.The incidence of retinopathy 10 years after diagnosis in young adult people with diabetes. Diabetes Care 2003;26:349-354

21. Jenkins D, Kendall C, McKeown-Eyssen G, Josse RG, Silverberg J, Booth GL, et al. Effect of a Low-Glycemic Index or a High-Cereal Fiber Diet on Type 2 Diabetes. JAMA, 2008;300(23): 2742-2753.

22. King G, Gakidou E, Ravishankar N, Moore RT, Lakin J,Vargas M, et al.A 'Politically Robust' Experimental Design for Public Policy Evaluation, with Application to the Mexican Universal Health Insurance Program. Journal of Policy Analysis and Management 2007;26(3):479-506. [Consultado diciembre 26, 208] Disponible en: http://gking.harvard.edu/files/abs/spdabs.shtml

23. Aguilar-Salinas CA, Velazquez-Monroy O, Gómez-Pérez FJ, Gonzalez Chávez AG, Esqueda AL, Molina Cuevas V, et al. Characteristics of patients with type 2 diabetes in México. Diabetes Care 2003;26(7):202I-2026. 24. Gudbjornsttir S, Cederholm J, Nilsson PM, Eliasson B.An implementation of the St.Vincent Declaration for quality improvement in diabetes care. Diabetes Care 2003;26:1270-1276.

25.Coon P, Zulkowski K. Adherence to American Diabetes Association Standards of Care by rural health providers Diabetes Care 2002; 25:2224-2229.

26. Ruelas GV, Roybal M, LuY, Goldman D, Peters A. Clinical and Behavioral Correlates of Achieving and Maintaining Glycemic Targets in an Underserved Population With Type 2 Diabetes. Diabetes Care 2009:32:54-56.

27. Nettleton JA, Steffen L, Ni H, Liu K, Jacobs DR Jr. Dietary Patterns and Risk of Incident Type 2 Diabetes in the Multi-Ethnic Study of Atherosclerosis (MESA). Diabetes Care 2008; 31:1777-I782. 28. Sigal RJ, Kenny GP,Wasserman DH, Castaneda-Sceppa C. Physical activity/Exercise and type-2 diabetes. Diabetes Care 2004;27:25 I8-2539. 29. Mainous AG, Diaz VA, Saxena S, Baker R, Everett CJ, Koopman RJ, et al. Diabetes management in the USA and England: comparative analysis of national surveys. J R Soc Med 2006;99: 463-469. 